AUTORES:

Lorenzo I. Laporta ${ }^{1}$

José Afonso ${ }^{1}$

Isabel Mesquita $^{1}$

${ }^{1}$ Centro de Investigação Formação Inovação e intervenção em Desporto (CIFI2D), Faculdade de Desporto, Universidade do Porto, Portugal

https://doi.org/10.5628/RPCD.18.03.33
Contributo da análise

de redes sociais para

a análise do jogo:

Breve sinopse concetual

e sinóptica

\section{PLAVRAS CHAVE:}

Análise da performance. Análise do jogo.

Centralidade de autovetor. Ações de jogo.

Análise sistémica.

SUBMISSÃO: 25 de Outubro de 2018

ACEITAÇÃO: 20 de Dezembro de 2018

\title{
RESUMO
}

Na tentativa de melhor responder aos complexos problemas emergentes da performance desportiva, a análise de jogo vem explorando diversas metodologias. De entre estas, a análise de redes sociais vem ocupando um espaço crescente na análise de jogo aplicada aos jogos desportivos, que podem ser analisados com redes de interação. Com raiz matemática na teoria dos grafos, a análise de redes sociais permite analisar as relações entre diferentes unidades de análise. As pesquisas em desporto têm utilizado, maioritariamente, os jogadores enquanto unidade de análise (nós). Recentemente, verificou-se uma promissora expansão para a análise de ações de jogo enquanto nós. Contudo, pela complexidade de interações entre as unidades de análise e suas consequências, as relações indiretas entre estas deverão ser ponderadas e, neste sentido, a escolha da métrica mais apropriada é decisiva. A análise de redes sociais alcança este propósito através da centralidade de autovetor, cuja utilização vem emergindo no contexto de jogos desportivos. Todavia, as pesquisas tendem a analisar somente certas fases ou momentos do jogo ou, alternativamente, condensando-os, e, com isso, perdendo informação acerca da sua especificidade. Em suma, apesar das promissoras avenidas, carece a aplicação duma abordagem sistémica para analisar as inter-relações do jogo na sua complexidade e especificidade funcional. 


\title{
Contribution of social network analysis \\ to game analysis: Brief conceptual \\ and synoptic synopsis
}

\begin{abstract}
In an attempt to better respond to the complex problems emerging from sports performance, game analysis has been exploring several methodologies. Among these, social network analysis has been occupying a growing space in the game analysis applied to the sports games, which can be analyzed with Interaction Networks. With a mathematical root in graph theory, social networks analysis allows analyzing relations between different analysis units. Sports research have mostly used players as the analysis unit (nodes). Recently, there has been a promising expansion for the analysis of game actions while nodes. However, because of the interactions' complexity between the analysis units and their consequences, the indirect relationships between them should be weighed and, in this sense, the choice of the most appropriate metric is decisive. Social network analysis achieves this purpose through the autovector centrality, whose use has been emerging in the context of sports games. However, surveys tend to analyze only certain phases or game moments or, alternatively, condense them and thereby lose information about their specificity. In short, in spite of the promising avenues, it is necessary to apply a systemic approach to analyze the interrelationships of the game in its complexity and functional specificity.
\end{abstract}

\section{KEYWORDS:}

Performance analysis. Game analysis. Eigenvectors centrality. Game actions. Systemic analysis. 
A análise da performance nos jogos desportivos (JD) constitui um tema profundamente complexo e que tem motivado uma diversidade correspondente de abordagens, da qual iremos destacar a análise do jogo (AJ). A AJ busca analisar o fenómeno da performance desportiva de diferentes óticas. Nos últimos anos, as abordagens sistémicas, respeitadoras da complexidade dos JD, vêm assumindo um lugar de relevo (Lames \& McGarry, 2007; Lebed, 2006; McGarry, Anderson, Wallace, Hughes, \& Franks, 2002; Thelen, 2005). Todavia, a representação e avaliação das múltiplas interações entre inúmeras variáveis não constitui um problema de fácil resolução. Neste âmbito, distintas metodologias vêm tentando capturar esta complexidade, como, por exemplo, análises descritivas (Castro \& Mesquita, 2010), qui-quadrado (Laporta, Nikolaidis, Thomas, \& Afonso, 2015), análises preditivas (Marcelino, Mesquita, \& Sampaio, 2011), a regressão logística multinominal (Afonso \& Mesquita, 2011), a análise da função discriminante (Sampaio, Lago, Gonçalves, Maçãs, \& Leite, 2014), a análise sequencial de retardos (Afonso, Mesquita, Marcelino, \& Silva, 2010) e a análise de clusters (Sankaran, 2014).

Neste contexto, a análise de redes sociais (ARS) constitui uma poderosa e promissora ferramenta para auxiliar na resposta aos problemas abordados pela AJ. Emergente da psicologia e da sociologia (Freeman, 2004), o seu objetivo primordial consiste em desvendar os comportamentos dos atores, emergentes das suas interações (Borgatti, Everett, \& Johnson, 2013; Wasserman \& Faust, 1994). Assim, alicerçada na teoria dos grafos (Wäsche, Dickson, Woll, \& Brandes, 2017), são verificados padrões estatísticos de interação do comportamento entre elementos de um sistema (Borgatti et al., 2013; Freeman, 2004; Quatman \& Chelladurai, 2008a; Wasserman \& Faust, 1994). A utilização da ARS nas ciências do desporto vem assumindo maior preponderância nas últimas décadas, pois auxilia na busca de padrões nos fluxos de jogo (Wäsche et al., 2017).

A ARS possibilita a consideração de diferentes tipos de unidades de análise. Apesar dos jogadores constituírem a unidade de análise mais usual nas pesquisas com ARS em desporto (e.g., Clemente, Martins, Kalamaras, \& Mendes, 2015; Malta \& Travassos, 2014; Sasaki, Yamamoto, Miyao, Katsuta, \& Kono, 2017), vem-se observando uma expansão do tipo de unidades de análise utilizados neste contexto (e.g., Hurst et al., 2016, 2017; Loureiro et al., 2017). Conforme iremos analisar, tais abordagens vêm expandindo o âmbito de utilização das ferramentas da ARS no desporto, nomeadamente incrementando a profundidade $e$ diversidade de perspetivas de tratamentos conferidos a problemas emergentes.

Além das diferentes possibilidades de escolha relativamente à unidade de análise, a ARS engloba um conjunto alargado de ferramentas matemáticas, permitindo a seleção de diferentes métricas (Freeman, 1978). Cada métrica apresenta potencialidades e limitações distintas, pelo que a sua escolha deverá estar profundamente dependente do problema de estudo. No âmbito das ciências do desporto, as métricas de centralidade realçam os agen- 
tes mais influentes em determinado conjunto de interações (Borgatti, 2005; Ramos, Lopes, \& Araújo, 2017; Ribeiro, Silva, Duarte, Davids, \& Garganta, 2017). Todavia, a maioria destas métricas apenas considera as ligações diretas entre unidades de análise (e.g., centralidade de grau [Borgatti 2005; Freeman, 1978]). Existem, porém, métricas que consideram as ligações indiretas no estabelecimento dos pesos de centralidade atribuídos a cada unidade de análise (e.g., centralidade de autovetor [Bonacich, 2007; Bonacich \& Lloyd, 2001]).

0 presente ensaio visa elucidar o enfoque que vem sendo dado na utilização da ARS nos JD, realçando potenciais avenidas de pesquisa ainda por explorar. Em particular: (a) perscrutando a potencial expansão da tipologia de unidades de análise adotada; (b) analisando as potencialidades de se pesarem as interações indiretas em adição às diretas, na busca duma análise mais completa dos fenómenos investigados; e (c) sugerindo caminhos de relevo a trilhar por investigações futuras.

\section{CONCEITOS BASILARES DA ANÁLISE DE REDES SOCIAIS}

\section{SINOPSE HISTÓRICA E TIPOLOGIA DE REDES}

Alicerçada na matemática formal, a ARS emergiu na década de 1930, advinda da sociologia e psicologia e impulsionada pelos desenvolvimentos metodológicos e computacionais (Freeman, 2004). Baseada em técnicas e métricas adaptadas da teoria dos grafos (Wäsche et al., 2017), a ARS permite descobrir padrões de interação do comportamento relacional entre atores (Freeman, 2004; Quatman \& Chelladurai, 2008a), auxiliando na compreensão da sua estrutura, função, interação e dinâmica destes elementos dentro de um sistema (Passos et al., 2011). A raiz matemática da teoria dos grafos possibilita expandir as unidades de análise da ARS, que não necessita de ficar limitada a agentes, conforme será explorado neste ensaio. Desta forma, através de uma perspetiva relacional, torna-se pertinente a utilização desta metodologia no campo desportivo (Grund, 2012; Ribeiro et al., 2017; Wäsche et al., 2017).

Diversos tipos de redes podem ser estabelecidos com recurso à ARS. Num trabalho seminal, Wäsche et al. (2017) organizaram uma categorização da literatura existente a respeito da ARS no desporto. Os resultados permitiram desenvolver uma tipologia conceitual deste campo de pesquisa, elucidando seis tipos diferentes de redes utilizadas: redes de competição, redes de interorganização, redes de intraorganização, redes de afiliação, ambientes sociais e redes de interação.

As redes de competição são caracterizadas pela análise de diferentes eventos (intereventos), onde os padrões estruturais e o desempenho de atletas ou equipes desportivas são fornecidos através de resultados de jogos, competições, entre outros. Os autores ainda mencionam que uma possibilidade de aplicação da ARS poderia ser o cálculo de probabilidades de apostas (ver Bothner, Kim, \& Smith, 2012; Breznik \& Batagelj, 2012; Jessop, 2006; Mukherjee, 2012; Radicchi, 2011; Saavedra, Powers, McCotter, Porter, \& Mucha, 2010; Sanders, 2011). 
Redes de interorganização analisam as relações estruturais entre organizações, como, por exemplo, associações desportivas, ligas ou clubes. Os autores subdividiram em dois domínios: gestão desportiva e literatura relacionada ao desporto. Os estudos relacionados ao gerenciamento desportivo levaram em conta a relação entre entidades desportivas (ver Cobbs, 2011; Cousens, Barnes, \& MacLean, 2012; Sallent, Palau, \& Guia, 2011; Seevers, Skinner, \& Dahlstrom, 2010), enquanto que a literatura desportiva ${ }^{1}$ relacionou os artigos científicos através das citações destes estudos (ver Agulló-Calatayud, González-Alcaide, Valderrama-Zurián, \& Aleixandre-Benavent, 2008; Bruner, Erickson, Wilson, \& Côté, 2010; Love \& Andrew, 2012; Quatman \& Chelladurai, 2008b).

Já as redes de intraorganização revelam as características de dentro das organizações. Apesar dos poucos estudos nesta temática, eles revelaram aspetos como conflitos sociais, relações de amizade e de confiança, eficácia dos membros de um clube, o relacionamento destes aspetos com o impacto no desempenho desportivo (Warner, Bowers, \& Dixon, 2012; Zachary, 1977). Além disso, redes onde há trocas de informações táticas, informações a respeito da eleição de presidentes e conselheiros desportivos, também fazem parte deste tipo de redes.

As redes de afiliação compreendem os dois tipos de redes citadas anteriormente (intra e interorganizacionais), pois consideram indivíduos nas organizações e a participação de atores em eventos. Além disso, pode-se incluir uma rede que leve em conta a diretoria de um clube desportivo, bem como redes corporativas relacionadas com patrocínios e com a interatividade entre atletas (Hambrick, 2012).

Ambientes sociais são redes dedicadas aos ambientes nos quais os indivíduos estão inseridos. No caso do desporto, as redes sociais online (e.g., twitter) influenciam o comportamento dos atores e são também moldadas por eles, considerando a comunicação dos organizadores de eventos desportivos e de seus seguidores (ver Hambrick, 2012). Além disso, a ARS pode ser aplicada para entender a influência do ambiente social na participação desportiva, no doping ou nas lesões dos atletas.

Por fim, as redes de interação, diferentemente das redes de competição, consideram a interação entre jogadores dentro de uma equipe, isto é, são caracterizadas por conterem relações baseadas nas regras do jogo e no desempenho dos jogadores dentro de uma equipe, como por exemplo a relação dos atletas através dos passes realizados no futebol (Duch, Waitzman, \& Amaral, 2010; Grund, 2012), no basquetebol (Fewell, Armbruster, Ingraham, Petersen, \& Waters, 2012) e em jogadores de polo aquático (Passos et al., 2011). Os autores reforçam quem este tipo de rede e as redes de competição são específicas no âmbito desportivo, enquanto que as outras são importantes para outros contextos sociais com os mesmos tipos de nós e relações.

\footnotetext{
${ }^{1}$ Os autores mencionam que este tipo de categorização poderia ser um novo tipo de rede, porém consideraram como redes interorganizacionais, pois os grupos de autores, ou autores individuais, são também organizações sociais.
} 


\section{UNIDADES DE ANÁLISE E MÉTRICAS}

No contexto da ARS, as unidades básicas de observação de uma rede são designadas por nós (Brass, Galaskiewicz, Greve, \& Tsai, 2004). Os nós relacionam-se entre si, formando ligações que são designadas por arestas (Wäsche et al., 2017). As arestas podem representar distintos tipos de relações determinadas pelo problema de estudo. Por exemplo, os jogadores podem-se relacionar através dos passes, os funcionários de uma empresa através de relações de empatia, as células pela interação molecular, entre outros exemplos (Barabasi \& Oltvai, 2004; Bode, Wood, \& Franks, 2011; Passos et al., 2011). No conjunto, os nós e arestas fornecem características do comportamento de todo ambiente (Freeman, 2004; Quatman \& Chelladurai, 2008a).

Embora a ARS tenda a considerar os agentes como unidades de análise (Grund, 2012; Ribeiro et al., 2017; Wäsche et al., 2017), a sua raiz na teoria dos grafos possibilita a exploração de unidades de análise não-agenciais. Por exemplo, segundo Ortiz-Pelaez, Pfeiffer, Soares-Magalhães e Guitian (2006), a definição de elementos e suas relações depende da questão a ser pesquisada e respondida, pois os nós podem assumir diversas tipologias (e.g., atletas, propriedades, animais, vizinhanças). Com efeito, alguns pesquisadores ampliaram já a utilização desta ferramenta e consideraram a interconexão de ações ${ }^{2}$ como nós e a relação entre estas ações como as arestas (Afonso, Laporta, \& Mesquita, 2017; Hurst et al., 2016, 2017; Loureiro et al., 2017). De resto, o valor de centralidade não considera o tipo de unidade de análise selecionada, mas somente a ponderação das inter-relações estabelecidas.

A ASR apresenta um diversificado conjunto de métricas, cada uma com potencialidades e limitações específicas. Deste modo, uma clara definição do problema de estudo é essencial, determinando o tipo de métricas mais ajustáveis. Destaque para as métricas de centralidade, que avaliam a posição ou a importância funcional dos elementos, e, dependendo da medida, podem estar associadas a diferentes vantagens ou desvantagens para os atores (Wäsche et al., 2017; Zuo et al., 2011). Estas métricas revelam a importância de cada unidade de análise (nó) na rede (Borgatti, 2005). De entre esta classe de métricas, quatro centralidades são comumente utilizadas: grau, intermediação, proximidade e autovetor (Freeman, 1978; Ribeiro et al., 2017).

A centralidade de grau considera o número de ligações diretas entre nós. A centralidade de intermediação pesa o número de vezes que um nó se conecta a dois outros nós através de seus caminhos mais curtos. A centralidade de proximidade considera o somatório das distâncias de todos os caminhos mais curtos de um nó a outro em um gráfico. Destaca-se a centralidade de autovetor (eigenvector) de Bonacich $(1987,2007)$, conhecida como uma medida de status (Bonacich \& Lloyd, 2001), a qual prevê que as trajetórias podem

\footnotetext{
${ }^{1}$ Ações de jogo foram consideradas como a unidade básica de análise em cada complexo de jogo; assim, os jogadores realizam-nas na busca de marcar um ponto, ou dificultar as ações adversárias.
} 
revisitar nós e arestas várias vezes ao longo do caminho (Borgatti, 2005). Assim, enquanto a métrica de grau, por exemplo, considera a contagem do número de nós adjacentes a ele (Wasserman \& Faust, 1994), a de autovetor pondera as conexões diretas e indiretas que chegam a um nó (Bonacich, 1987, 2007; Ramos et al., 2017; Wasserman \& Faust, 1994). Ou seja, a centralidade de autovetor pondera os pesos atribuídos não somente às ligações diretas entre unidades de análise, mas também as ligações indiretas, considerando interações de $n$-ordem (Bonacich, 2007; Bonacich \& Lloyd, 2001). Neste sentido, esta medida torna-se uma poderosa ferramenta para o desporto, pois pode realçar o papel relativo de cada nó dentro do sistema de jogo, após ter pesado todas as suas ligações, sejam diretas ou indiretas (Afonso et al., 2017; Hurst et al., 2016, 2017; Loureiro et al., 2017).

\section{SINOPSE DE INVESTIGAÇÃO CENTRADA NA ANÁLISE DE REDES SOCIAIS EM JOGOS DESPORTIVOS}

\section{JOGADORES COMO UNIDADE DE ANÁLISE E MÉTRICAS USUAIS}

A investigação com recurso à ARS em ciências do desporto tem ocorrido, precisamente, mais no âmbito da AJ. Neste contexto, as pesquisas têm recorrido, de forma quase exclusiva, aos jogadores como unidade de análise (os nós da ARS) e aos passes como ligações entre essas unidades (as arestas da ARS).

No futebol, alguns autores, considerando jogadores como nós e o passe entre eles como arestas, revelaram padrões na construção ofensiva. Malta e Travassos (2014), através das centralidades de intermediação e de grau (entrada e saída), revelaram que a equipe analisada (pertencente à Primeira Liga Portuguesa) possuía padrões preferenciais de transição defesa-ataque, onde as decisões do tipo de passe utilizado dependiam do número de participantes que cercavam a bola. Alves, Gonçalo, José, Vasco e Miguel (2018) analisaram uma seleção de futebol (sub-20), onde os jogadores médio-centro e defesa-esquerdo fora, através da centralidade de grau, foram os atletas mais centrais da rede, enquanto que o médio-centro, através da centralidade de autovetor, foi o jogador-chave na dinâmica coletiva ofensiva da equipa até 0 golo. Na mesma linha, Gama et al. (2014) observaram seis jogos da Primeira Liga Portuguesa de futebol e constataram que alguns jogadores foram peças-chave na construção do ataque, assumindo um papel de elevada centralidade e importância na dinâmica da equipe.

Apesar da maioria dos estudos terem sido aplicados no futebol, a ARS já foi utilizada em outros JD, como o basquetebol. Clemente, Martins, Kalamaras e Mendes (2015) verificaram a contribuição individual dos jogadores das diferentes posições táticas no processo de ataque em equipas de diferentes níveis de competição (sub-14, sub-16, sub-18 e amadores com mais de 20 anos). Apesar de não encontrarem diferenças entre as posições táticas nos diferentes níveis competitivos, encontraram entre a centralidade de grau de entrada e saída, onde o armador, em particular, obteve um papel de destaque e de ligação entre os 
demais jogadores, recebendo e passando a bola na construção ofensiva. No cricket, Dey, Ganguly e Roy (2017) objetivaram formar uma equipa internacional de jogadores pertencentes à Liga Principal Indiana de 2016. As centralidades de intermediação, proximidade e grau indicaram que 11 jogadores tiveram melhor desempenho e mereciam pertencer à equipa. Porém, os autores concluíram que outros aspetos mereciam atenção, como a atuação contra um oponente forte, ou o desempenho e companheirismo com um membro efetivo de equipe, entre outros.

No voleibol, um dos primeiros estudos realizados foi o de Clement et al. (2015), que verificaram a variação de centralidade de grau (entrada e saída) e pagerank entre as redes de duas equipes de diferentes níveis (com idade abaixo de 12 anos e amadores) do Campeonato Regional Português de voleibol. Apesar de não existirem diferenças significativas entre os dois níveis analisados, os jogadores de maior e menor nível de proeminência foram da zona 3 e 5, respetivamente; o primeiro deve-se ao fato de que, nas duas equipes analisadas, o primeiro toque era sempre direcionado para o jogador desta zona na fase de ataque, enquanto que no segundo esta zona foi associada a menor eficácia de ataque.

Um outro estudo em futebol apresentou já alguma inovação relativamente às métricas, embora não relativamente à unidade de análise. Aquino et al. (2018) analisaram 18 partidas da $3^{a}$ divisão do futebol brasileiro, através do efeito de variáveis situacionais (i.e., estágio da competição, localização da partida, qualidade de oposição e resultado do jogo), formação de equipe adversária, desempenho de corrida a partir das posições de jogo (GPS), e, ainda, a interação da equipe utilizando a ARS. Dentre os principais resultados, os jogadores meio-campistas centrais/ externos se encontravam mais próximos de outros companheiros (maior centralidade de proximidade), "controlavam" mais redes (elevada centralidade de intermediação), realizavam mais passes (grau de saída) e foram um jogador-chave na organização ofensiva da equipa (maior centralidade de autovetor) em comparação com os defensores centrais, externos e os atacantes. Aqui, temos o primeiro vislumbre de ponderação de ligações indiretas, com utilização do autovetor, mas ainda de forma tímida.

Também no râguebi, Sasaki t al. (2017) mostraram os turnovers realizados durante as ações defensivas na Copa do Mundo de Râguebi 2015 através da centralidade de autovetor, revelando que um maior desempenho na rotatividade da defesa contribuiu para a vitória. Em suma, as pesquisas utilizando ARS no desporto vêm utilizando quase exclusivamente os jogadores enquanto unidades de análise o que, sendo positivo, apenas providencia um quadro incompleto do fenómeno complexo que é a performance e, ademais, não utiliza todas as potencialidades oferecidas pela ARS.

\section{AÇÕES DE JOGO COMO UNIDADE DE ANÁLISE E PONDERAÇÃO DE LIGAÇÕES INDIRETAS}

Mais recentemente, as pesquisas vêm adotando um paradigma diferenciado, expandindo as potencialidades da ARS no desporto: (a) adotando as ações de jogo como unidades de análise; e (b) recorrendo à centralidade de autovetor como forma de ponderar as ligações 
diretas e indiretas entre unidades de análise (e.g., Hurst et al., 2016, 2017; Loureiro et al., 2017). Estes estudos foram todos realizados no voleibol de alto nível. Hurst et al. (2016, 2017) analisaram o serviço (KO), side-out (KI), contra-ataque (KII) e contra-ataque do contra-ataque (KIII) no primeiro estudo, e a cobertura de ataque (KIV) e bola morta (KV) no segundo, na fase de grupos do Women's World Grand Prix 2015. Os resultados indicaram que jogar fora do sistema (off-system) foi central nos complexos analisados, principalmente em ações como condições de distribuição, zona e tempo de ataque. Para além disso, e apesar do KV liberar um número maior de jogadores, tornando a ação de ataque potencialmente vantajosa, o jogo mostrou-se mais lento (com tempos de ataque mais lentos) mesmo com melhores condições de distribuição.

Afonso et al (2017) averiguaram as diferenças entre o KII e KIII no voleibol feminino analisando a fase final do Grand Prix 2015. Tratar estes dois complexos da mesma forma pode fazer com que informações sejam perdidas ou tratadas de forma similar quando apresentam diferenças, como, por exemplo, melhores condições de distribuição, jogo mais lento (tempo de ataque 3) e menos zonas de ataque (4 e 2) no KIII, enquanto no KII há uma maior variabilidade das zonas de ataque (zonas 4, 2, 1 e 6 mais centrais).

Seguindo a lógica anterior, Loureiro et al. (2017) analisaram os complexos 0, I, II e III no voleibol masculino de alto nível (Copa do Mundo Masculina 2015) e, contrariamente ao voleibol feminino, os resultados indicaram que no KI as equipes jogaram em condições próximas das ideais (in-system), porém apresentaram reduzida variação em relação às zonas e tempos de ataque; já no KII, em piores condições que no KIII, apesar existir maior variabilidade do número de bloqueadores (triplo e duplo) e melhores condições de distribuição, o tempo de ataque foi lento, provavelmente pela necessidade de recuperação do bloqueio, dificultando assim a participação em ataque mais rápidos.

Em suma, estes estudos revelaram a pertinência e aplicabilidade de considerar unidades de análise não-agenciais como, por exemplo, as ações de jogo. Ademais, estes estudos buscaram uma abordagem sistémica do jogo (McGarry et al., 2002), procurando avaliar as diferentes fases do jogo e suas interações diretas e indiretas (Hurst et al., 2016, 2017; Loureiro et al., 2017).

\section{LIMITAÇÕES E POSSIBILIDADES DE FUTUROS ESTUDOS}

A AJ constitui uma poderosa abordagem à análise da performance. Neste contexto, a ARS vem emergindo como uma metodologia de análise com elevado potencial. No entanto, as pesquisas neste âmbito estão grandemente limitadas à utilização de unidades de análise agenciais (e.g., jogadores), dessa forma ficando por explorar unidades não-agenciais (e.g., ações de jogo) que conferem informação diferenciada relativamente aos padrões de jogo. A utilização de unidades não-agenciais é possível devido às raízes matemáticas da ARS (i.e., teoria dos grafos). Recentemente, esta questão vem sendo explorada por investiga- 
ções em voleibol de alto nível (Hurst et al., 2016, 2017; Loureiro et al., 2017), mas desconhecemos aplicações similares noutras modalidades.

Realce, ainda, para o facto de poucos estudos recorrerem à centralidade de autovetor que, contudo, apresenta um elevado potencial por pesar as ligações indiretas e não somente as diretas e, assim, efetuar cálculos ponderados que consideram um maior nível de complexidade e, porventura, aportam uma perspetiva mais profunda acerca dos fenómenos estudados. As recentes pesquisas em voleibol (Hurst et al., 2016, 2017; Loureiro et al., 2017) adotaram ações de jogo enquanto unidades de análise e o autovetor para pesagem de ligações indiretas. Contudo, não estudaram o jogo na sua globalidade, mas somente alguns dos seus complexos, carecendo, desta forma, de uma verdadeira visão sistémica.

Adicionalmente, não deixa de ser surpreendente que, sendo a ARS utilizada pela AJ como forma de analisar a performance, não haja, do nosso conhecimento, estudos que relacionem as ações de jogo com a eficácia das jogadas. Embora esta constitua uma prática comum na $A J$, quando recorre às mais diversas metodologias, tal abordagem ainda não foi concretizada neste contexto. Finalmente, muitos estudos, nas diferentes modalidades, situam-se no sexo masculino. Todavia, é reconhecido que, em muitos desportos, a performance é qualitativamente distinta, o que justificaria a necessidade de se estudarem os mesmos fenómenos nos dois sexos. Caso contrário, existe o risco de os resultados obtidos no masculino serem indevidamente extrapolados para o feminino. 
Afonso, J., Laporta, L., \& Mesquita, I. (2017). A importância de diferenciar o KII do KIII no voleibol feminino de alto nível. Revista Portuguesa de Ciências do Desporto, 17(S1.A), 45-58. doi:10.5628/rpcd.17.S1A.140

Afonso, J., \& Mesquita, I. (2011). Determinants of block cohesiveness and attack efficacy in high-level women's volleyball. European Journal of Sport Science, 11 (1) , 69-75. doi:10.1080/17461391.2010.487114 Afonso, J., Mesquita, I., Marcelino, R., \& Silva, J. A. (2010). Analysis of the setter's tactical action in high-performance women's volleyball. Kinesiology, 42(1), 82-89.

Agulló-Calatayud, V., González-Alcaide, G., Valderrama-Zurián, J. C., \& Aleixandre-Benavent, R. (2008). Consumption of anabolic steroids in sport, physical activity and as a drug of abuse: An analysis of the scientific literature and areas of research. British Journal of Sports Medicine, 42(2), 103-109. doi:10.1136/ bjsm.2007.036228

Alves, R., Gonçalo, D., José, G., Vasco, V., \& Miguel, C. (2018). Interação e network de sequências ofensivas coletivas: Análise de uma seleção de sub-20 no Campeonato do Mundo de Futebol. Revista Portuguesa de Ciências do Desporto, 16(3), 44-56. doi:10.5628/ RPCD.16.03.44

Aquino, R., Carling, C., Vieira, L., Martins, G., Jabor, G., Machado, J., ... Puggina, E. (2018). Influence of situational variables, team formation and playing position on match running performance and social network analysis of Brazilian professional soccer players. Journal of Strength and Conditioning Research. Advance online publication. doi:10.1519/ JSC.0000000000002725

Barabasi, A. L., \& Oltvai, Z. N. (2004). Network biology: Understanding the cell's functional organization. Nature Reviews Genetics, 5(2), 101-113. doi:10.1038/nrg1272

Bode, N. W., Wood, A. J., \& Franks, D. W. (2011). The impact of social networks on animal collective motion. Animal Behaviour, 82(1), 29-38. doi:10.1016/j. anbehav.2011.04.011

Bonacich, P. (1987). Power and centrality: A family of measures. American Journal of Sociology, 92(5), 1170-1182.

Bonacich, P. (2007). Some unique properties of eigenvector centrality. Social Networks, 29(4), 555-564. doi:10.1016/j.socnet.2007.04.002

Bonacich, P., \& Lloyd, P. (2001). Eigenvector-like measures of centrality for asymmetric relations. Social Networks, 23(3), 191-201.
Borgatti, S. P. (2005). Centrality and network flow. Social Networks, 27(1), 55-71. doi:10.1016/j.socnet.2004.11.008 Borgatti, S. P., Everett, M. G., \& Johnson, J. C. (2013). Analyzing social networks. Thousand Oaks, CA: SAGE. doi:10.1016/S0378-8733(01)00038-7

Bothner, M. S., Kim, Y. K., \& Smith, E. B. (2012). How does status affect performance? Status as an asset vs. status as a liability in the PGA and NASCAR. Organization Science, 23(2), 416-433. doi.org/10.1287/orsc.1110.0679

Brass, D. J., Galaskiewicz, J., Greve, H. R., \& Tsai, W. (2004). Taking stock of networks and organizations: A multilevel perspective. Academy of Management Journal, 47(6), 795-817. doi:10.5465/20159624

Breznik, K., \& Batagelj, V. (2012). Retired matches among male professional tennis players. Journal of Sports Science \& Medicine, 11 (2), 270-278.

Bruner, M. W., Erickson, K., Wilson, B., \& Côté, J. (2010). An appraisal of athlete development models through citation network analysis. Psychology of Sport and Exercise, 11(2), 133-139. doi:10.1016/j. psychsport.2009.05.008

Castro, J., \& Mesquita, I. (2010). Analysis of the attack tempo determinants in volleyball's complex II: A study on elite male teams. International Journal of Performance Analysis in Sport, 10(3), 197-206. doi:10 .1080/24748668.2010.11868515

Clemente, F. M., Martins, F. M. L., Kalamaras, D., \& Mendes, R. S. (2015). Network analysis in basketball: Inspecting the prominent players using centrality metrics. Journal of Physical Education and Sport, 15(2), 212-217. doi:10.7752/jpes.2015.02033

Clemente, F. M., Martins, F. M. L., \& Mendes, R. S. (2015). There are differences between centrality levels of volleyball players in different competitive levels? Journal of Physical Education and Sport, 15(2), 272-276. doi:10.7752/jpes.2015.02041

Cobbs, J. B. (2011). The dynamics of relationship marketing in international sponsorship networks. Journal of Business \& Industrial Marketing, 26(8), 590601. doi:10.1108/08858621111179868

Cousens, L., Barnes, M., \& MacLean, J. (2012). Strategies to increase sport participation in Canada: The role of a coordinated network. International Journal of Sport Management and Marketing, 12(3-4), 198216. doi:10.1504/IJSMM.2012.052667

Dey, P., Ganguly, M., \& Roy, S. (2017). Network centrality based team formation: A case study on T-20 cricket. Applied Computing and Informatics, 13(2), 161-168. doi:10.1016/j.aci.2016.11.001 
Duch, J., Waitzman, J. S., \& Amaral, L. A. N. (2010). Quantifying the performance of Individual Players in a team activity. PLoS ONE, 5(6), e10937. doi:10.1371/ journal.pone.0010937

Fewell, J. H., Armbruster, D., Ingraham, J., Petersen, A., \& Waters, J. S. (2012). Basketball teams as strategic networks. PLoS ONE, 7(11), e47445. doi:10.1371/ journal.pone.0047445

Freeman, L. C. (1978). Centrality in social networks conceptual clarification. Social Networks, I(3), 215239. doi:org/10.1016/0378-8733(78)90021-7

Freeman, L. C. (2004). The development of social network analysis: A study in the sociology of science (Vol. 1). North Charleston, SC: BookSurge. doi:10.1016/j. socnet.2005.06.004

Gama, J., Passos, P., Davids, K., Relvas, H., Ribeiro, J., Vaz, V., \& Dias, G. (2014). Network analysis and intra-team activity in attacking phases of professional football. International Journal of Performance Analysis in Sport, 14(3), 692-708. doi:10.1080/24748668.201 4.11868752

Grund, T. U. (2012). Network structure and team performance: The case of English Premier League soccer teams. Social Networks, 34(4), 682-690. doi:10.1016/j.socnet.2012.08.004

Hambrick, M. E. (2012). Six degrees of information: Using social network analysis to explore the spread of information within sport social networks. International Journal of Sport Communication, 5(1), 16-34. doi:10.1123/ijsc.5.1.16

Hurst, M., Loureiro, M., Valongo, B., Laporta, L., Nikolaidis, P. T., \& Afonso, J. (2016). Systemic mapping of high-hevel women's volleyball using social network analysis: The case of serve (KO), side-out (KI), side-out transition (KII) and transition (KIII). International Journal of Performance Analysis in Sport, 16 (2), 695-710. doi:10.1080/24748668.2016.11868917

Hurst, M., Loureiro, M., Valongo, B., Laporta, L., Nikolaidis, P., \& Afonso, J. (2017). Systemic mapping of high-level women's volleyball using social network analysis: The case of attack coverage, freeball and downball. Montenegrin Journal of Sports Science and Medicine, 6(1), 57-64.

Jessop, A. (2006). A measure of competitiveness in leagues: A network approach. Journal of the Operational Research Society, 57(12), 1425-1434. doi:10.1057/palgrave.jors.2602122

Lames, M., \& McGarry, T. (2007). On the search for reliable performance indicators in game sports. International Journal of Performance Analysis in Sport, 7(1), 62-79. doi:10.1080/24748668.2007.11868388
Laporta, L., Nikolaidis, P., Thomas, L., \& Afonso, J. (2015). The importance of loosely systematized game phases in sports: The case of attack coverage systems in high-level women's volleyball. Montenegrin Journal of Sports Science and Medicine, 4(1), 19-24.

Lebed, F. (2006). System approach to games and competitive playing. European Journal of Sport Science, 6(1), 33-42. doi:10.1080/17461390500422820

Loureiro, M., Hurst, M., Valongo, B., Nikolaidis, P., Laporta, L., \& Afonso, J. (2017). A comprehensive mapping of high-level men's volleyball gameplay through social network analysis: Analysing serve, side-out, side-out transition and transition. Montenegrin Journal of Sports Science and Medicine, 6(2), 35-41.

Love, A., \& Andrew, D. P. (2012). The intersection of sport management and sociology of sport research: A social network perspective. Sport Management Review, 15(2) , 244-256. doi:10.1016/j.smr.2011.08.001 Malta, P., \& Travassos, B. (2014). Caraterização da transição defesa-ataque de uma equipa de futebol. Motricidade 10(1), 27-37. doi:10.6063/motricidade.10(1).1544

Marcelino, R., Mesquita, I., \& Sampaio, J. (2011). Effects of quality of opposition and match status on technical and tactical performances in elite volleyball. Journal of Sports Sciences, 29(7), 733-741. doi:10.108 0/02640414.2011.552516

McGarry, T., Anderson, D., Wallace, S., Hughes, M., \& Franks, I. (2002). Sport competition as a dynamical self-organizing system. Journal of Sports Sciences, 20(10), 771-781.

Mukherjee, S. (2012). Identifying the greatest team and captain: A complex network approach to cricket matches. Physica A: Statistical Mechanics and its Applications, 391(23), 6066-6076. doi:10.1016/j.physa.2012.06.052

Ortiz-Pelaez, A., Pfeiffer, D., Soares-Magalhaes, R., \& Guitian, F. (2006). Use of social network analysis to characterize the pattern of animal movements in the initial phases of the 2001 foot and mouth disease (FMD) epidemic in the UK. Preventive Veterinary Medicine, 76(1-2), 40-55.

Passos, P., Davids, K., Araújo, D., Paz, N., Minguéns, J., \& Mendes, J. (2011). Networks as a novel tool for studying team ball sports as complex social systems. Journal of Science and Medicine in Sport, 14(2), 170176. doi:10.1016/j.jsams.2010.10.459

Quatman, C., \& Chelladurai, P. (2008a). Social network theory and analysis: A complementary lens for inquiry. Journal of Sport Management, 22(3), 338360. doi:10.1123/jsm.22.3.338 
Quatman, C., \& Chelladurai, P. (2008b). The social construction of knowledge in the field of sport management: A social network perspective. Journal of Sport Management, 22 (6), 651-676. doi:10.1123/jsm.22.6.651

Radicchi, F. (2011). Who is the best player ever? A complex network analysis of the history of professional tennis. PLoS ONE, 6(2), e17249. doi:10.1371/ journal.pone.0017249

Ramos, J., Lopes, R. J., \& Araújo, D. (2017). What's next in complex networks? Capturing the concept of attacking play in invasive team sports. Sports Medicine, 48(1), 17-28. doi:10.1007/s40279-017-0786-z

Ribeiro, J., Silva, P., Duarte, R., Davids, K., \& Garganta, J. (2017). Team sports performance analysed through the lens of social network theory: Implications for research and practice. Sports Medicine, 47(9), 16891696. doi:10.1007/s40279-017-0695-1

Saavedra, S., Powers, S., McCotter, T., Porter, M. A., \& Mucha, P. J. (2010). Mutually-antagonistic interactions in baseball networks. Physica A: Statistical Mechanics and its Applications, 389(5), 1131-1141. doi:10.1016/j.physa.2009.10.038

Sallent, O., Palau, R., \& Guia, J. (2011). Exploring the legacy of sport events on sport tourism networks. European Sport Management Quarterly, 11(4), 397-421. doi:10.1080/16184742.2011.599208

Sampaio, J. E., Lago, C., Gonçalves, B., Maçãs, V. M., \& Leite, N. (2014). Effects of pacing, status and unbalance in time motion variables, heart rate and tactical behaviour when playing 5-a-side football small-sided games. Journal of Science and Medicine in Sport, 17(2), 229-233. doi:10.1016/j.jsams.2013.04.005

Sanders, J. (2011). Ranking patterns in college football's BCS selection system: How conference ties, conference tiers, and the design of BCS payouts affect voter decisions. Social Network, 33(4), 273280. doi:10.1016/j.socnet.2011.08.001

Sankaran, S. (2014). Comparing pay versus performance of IPL bowlers: An application of cluster analysis. International Journal of Performance Analysis in Sport, 14(1), 174-187. doi:10.1080/24748 668.2014.11868713

Sasaki, K., Yamamoto, T., Miyao, M., Katsuta, T., \& Kono, I. (2017). Network centrality analysis to determine the tactical leader of a sports team. International Journal of Performance Analysis in Sport, 17(6), 822831. doi:10.1080/24748668.2017.1402283

Seevers, M. T., Skinner, S. J., \& Dahlstrom, R. (2010). Performance implications of a retail purchasing network: The role of social capital. Journal of Retailing, 86(4), 310-321. doi:10.1016/j.jretai.2010.07.002
Thelen, E. (2005). Dynamic systems theory and the complexity of change. Psychoanalytic Dialogues, 15(2), 255-283. doi:10.1080/10481881509348831

Warner, S., Bowers, M. T., \& Dixon, M. A. (2012). Team dynamics: A social network perspective. Journal of Sport Management, 26(1), 53-66. doi:10.1123/ jsm.26.1.53

Wäsche, H., Dickson, G., Woll, A., \& Brandes, U. (2017). Social network analysis in sport research: An emerging paradigm. European Journal for Sport and Society, 14(2), 138-165. doi:10.1080/16138171.2017.1318198 Wasserman, S., \& Faust, K. (1994). Social network analysis: Methods and applications (Vol. 8). Cambridge, UK: Cambridge University Press.

Zachary, W. W. (1977). An information flow model for conflict and fission in small groups. Journal of Anthropological Research, 33(4), 452-473. doi:10.1086/ jar.33.4.3629752

Zuo, X. N., Ehmke, R., Mennes, M., Imperati, D., Castellanos, F. X., Sporns, O., \& Milham, M. P. (2011). Network centrality in the human functional connectome. Cerebral Cortex, 22(8), 1862-1875. doi:10.1093/cercor/bhr269

\section{2}

